\title{
Lung Injury Resulting from Fluted Silastic Soft Drain Extraction under Negative Pressure Suction*
}

\author{
Susumu Isoda, Tamizo Kimura, Kenji Nishimura, Nozomu Yamanaka, Shingo Nakamura, \\ Tadaaki Maehara
}

Department of Cardiovascular Surgery, National Defense Medical College, Tokorozawa, Japan.

Email: isodas@ndmc.ac.jp

Received June $4^{\text {th }}$, 2013; revised July $3^{\text {rd }}, 2013$; accepted July $17^{\text {th }}, 2013$

Copyright (C) 2013 Susumu Isoda et al. This is an open access article distributed under the Creative Commons Attribution License, which permits unrestricted use, distribution, and reproduction in any medium, provided the original work is properly cited.

\begin{abstract}
We experienced two cases of lung injury resulting from fluted silastic drain extraction under reservoir-generated negative pressure suction. In the first case, a 67-year-old man underwent coronary artery bypass grafting. A 19 Fr BLAKE drain was placed at the pericardial cavity, the mediastinum, and the left thoracic cavity. All three drains were connected to J-VAC reservoirs. After removing the drains (which maintained the negative pressure), subcutaneous emphysema and hemopneumothorax occur. A trocar catheter was inserted. Bleeding and the air leak terminated within a day. In the second case, a 73-year-old man underwent aortic valve replacement. Right pneumothorax occurred after the removal of the pleural cavity drain, which maintained the negative pressure generated by the reservoir. We inserted a trocar catheter, and the air leak terminated within a day. We hypothesized that the reservoir-generated negative pressure causes the pleura to enter the groove of the drain and become damaged during extraction. We stopped using a reservoir to connect to the fluted silastic drain placed at the pleural cavity and removed the negative pressure when extracting the pleural cavity drain. Since implementing this change, we have not experienced a similar lung injury in more than 500 cardiac surgery patients.
\end{abstract}

Keywords: Complication; Extraction; Lung Injury; Negative Pressure; Silastic Drain

\section{Introduction}

Flexible fluted small silicone drains have recently been used as chest drains after cardiac surgery [1-3]. In 2002, after Payne et al. [2] introduced the use of the silicone drain after coronary bypass surgery (using the internal mammary artery) and had good results, we introduced the use of the BLAKE drain (Ethicon, Tokyo Japan) and J-VAC reservoir (Ethicon, Tokyo Japan) to drain the pericardial cavity, mediastinal cavity, and pleural cavity. We experienced two cases of lung injury after silicone drain extraction under negative pressure suction generated by the J-VAC reservoir. This lung injury has not been reported elsewhere.

\section{Case Reports}

\section{Case 1}

A 67-year-old man experienced chest pain. He was admitted to our hospital and diagnosed as having an acute

\footnotetext{
*Susumu Isoda and other authors have no conflict of interest.
}

myocardial infarction. His creatine kinase level was 1315 $\mathrm{IU} / \mathrm{mL}$. Coronary angiography revealed $75 \%$ stenosis of the left main trunk, $75 \%$ stenosis of the left anterior descending artery, and $75 \%$ stenosis of the circumflex artery. The day after the angiography, the patient underwent off-pump coronary artery bypass grafting. The left internal mammary artery was anastomosed to the left anterior descending artery, and the radial artery was anastomosed to the ascending aorta and to the posterolateral branch. The left pleural cavity was opened at the internal mammary artery harvesting without intention. A BLAKE silicone drain (Hubless $19 \mathrm{Fr}$ ) was placed at the pericardial cavity, the mediastinum, and the left thoracic cavity. All three drains were connected to a J-VAC standard type reservoir $(300 \mathrm{~mL}$ closed-wound drainage reservoir). The pericardial and mediastinal drains were connected to one J-VAC reservoir. A second reservoir was connected to the chest drain. When we removed all drains on postoperative day 2 , the J-VAC reservoir was generating a negative pressure, which was estimated as more than $-40 \mathrm{mmHg}$. There was no resistance at the 
time of extracting the drain. Soon after removing the drains, the patient developed subcutaneous emphysema. Hemopneumothorax was subsequently diagnosed by chest radiography. A 30 Fr trocar catheter was inserted into the left thorax, and $350 \mathrm{~mL}$ of bloody effusion was removed. Bleeding stopped within a day. An air leak was initially noticed; however, it stopped within a day. The trocar catheter was removed 4 days after its insertion. His postoperative course was thereafter uneventful.

\section{Case 2}

A 73-year-old man with exertional dyspnea was diagnosed as having aortic insufficiency. His aortic valve was replaced by a 21-mm artificial valve (Carpentier-Edwards valve; Edwards Lifescience Co., Tokyo, Japan). His right chest was opened without intention. Three BLAKE (19 Fr) drains were placed at the pericardial cavity, mediastinal space, and right pleural cavity. The BLAKE drains were connected to a J-VAC reservoir. On the postoperative day 2, the pleural drain was removed without any resistance, while maintaining the negative pressure generated by the J-VAC standard reservoir. After removing all drains, the patient experienced a right pneumothorax. We inserted a 22 Fr trocar catheter into the right thorax. Bleeding was not apparent. One day after the trocar insertion, the sustained air leak stopped. The trocar catheter was removed two days after its insertion. The patient's course was thereafter uneventful.

\section{Discussion}

Mediastinal drainage and pleural drainage are necessary after cardiac surgical procedures to monitor bleeding and to prevent tamponade, hemothorax, or pneumothorax [4]. The use of a large bore rigid polyvinyl chloride chest tube has been the standard procedure. Fluted small soft silastic drains were newly introduced one decade ago and have been proven equally effective for draining the mediastinum and pleural cavities [5,6]. These new drains seem to cause less pain, are more comfortable, allow earlier ambulation, produce a smaller scar, and have less capacity for air evacuation $[1,7,8]$.

On searching previous reports in the medical literature, we could not find lung injury after the extraction of a fluted small silastic drain [1-3]. After we experienced these two cases of lung injury resulting from silastic drain extraction under negative pressure suction, we hypothesized that the strong negative pressure created by the standard type J-VAC reservoir causes the pleura to become attached to the fluted silicone drain and enter into the ditch of the silicone drain; therefore, extracting the fluted silastic drain without removing the negative pressure can tear pleural and lung tissue. We also considered that the long groove of the fluted silicon contains a long strip of pleura. This may carry a greater risk of injury than does the classic hard drain, which has holes that can not hold a large amount of pleural tissue. We have never experienced similar lung damage in 600 open heart surgery patients, even when we have extracted a classic hard pleural drain while maintaining a negative pressure. On the other hand, we had been using fluted small soft silastic drains and the J-VAC reservoir in approximately 200 open heart surgery cases before the two cases discussed in this paper. Therefore, we surmised that we needed to change the maneuver for extracting the pleural fluted silastic drain. To control pleural drainage, we stopped using the standard J-VAC standard reservoir, which generates a negative pressure over $-40 \mathrm{mmHg}$ and is not fully controllable. We returned to using a conventional system with three chambers: 1) the drainage chamber; 2) the water seal chamber; and 3) the suction control chamber (Q-1; N.Y.B. Co. Ltd, Atsugi, Japan). We set the negative pressure at $-10 \mathrm{~cm} \mathrm{H}_{2} \mathrm{O}$. To extract the pleural silicone drain, we completely stopped the negative pressure, clamped the drain, and removed it from the body gently.

To control pericardial and mediastinal drainage, we resumed using the conventional system with three chambers. If a patient does not have pleural drainage, the conventional system can be changed to a J-VAC reservoir for earlier ambulation of patients (i.e., 2 or 3 days after surgery). However, we are no longer as enthusiastic about it as previously because of the cost of the additional reservoir. If a patient has pleural drainage at a negative pressure of $-10 \mathrm{~cm} \mathrm{H}_{2} \mathrm{O}$, using the stronger J-VAC reservoir interferes with the conventional vacuum system. We are very confident when extracting a pericardial or mediastinal drain, but we suggest at least removing the strong negative force.

Since we changed the maneuver, we have performed more than 500 cardiac surgeries using 19 Fr fluted silicone drainage tubes and have had no complications of pneumothorax or hemopneumothorax after extracting the drain. We are now confident that our hypothesis may be correct that fluted silastic pleural drain extraction under negative pressure suction generated by the J-VAC reservoir can injure the lung.

\section{REFERENCES}

[1] J. A. Obney, M. J. Barrnes, P. G. Lisagor and D. J. Cohen, "A Method for Mediastinal Drainage after Cardiac Procedure Using Small Silastic Drains," The Annals of Thoracic Surgery, Vol. 70, No. 3, 2000, pp. 1109-1110. doi:10.1016/S0003-4975(00)01800-2

[2] M. Payne, G. J. Magovern Jr., D. H. Benckart, A. Vasilakis, G. W. Szydlowski, J. C. Cardone, et al., "Left pleural effusion after coronary artery bypass decreases with a supplemental pleural drain," The Annals of Thoracic Surgery, Vol. 73, No. 1, 2002, pp. 149-152. 
doi:10.1016/S0003-4975(01)03327-6

[3] T. L. Frankel, P. C. Hill, S. C. Stamou, R. C. Lowery, A. J. Pfister, A. Jain and P. J. Corso, "Silastic Drains vs Conventional Chest Tubes after Coronary Artery Bypass," Chest, Vol. 124, No. 1, 2003, pp. 108-113. doi:10.1378/chest.124.1.108

[4] E. R. Munnel and E. K. Thomas, "Current Concepts in Thoracic Drainage Systems," The Annals of Thoracic Surgery, Vol. 19, No. 3, 1975, pp. 261-268. doi:10.1016/S0003-4975(10)64016-7

[5] H. Niinami, M. Tabata, Y. Takeuchi and M. Umezu, "Experimental Assessment of the Drainage Capacity of Small Silastic Chest Drains," Asian Cardiovascular and Thoracic Annals, Vol. 14, No. 3, 2006, pp. 223-226. doi:10.1177/021849230601400311
[6] H. Ishikawa and S. Kimura, "The Flexible Silastic Drain after Chest Surgery: Novel Thoracic Drainage," The Annals of Thoracic Surgery, Vol. 81, No. 1, 2006, pp. 331334. doi:10.1016/j.athoracsur.2005.05.102

[7] N. Sakakura, T. Fukui, S. Mori, S. Hatooka, K. Yokoi and T. Mitsudomi, "Fluid Drainage and Air Evacuation Characteristics of Blake and Conventional Drains after Pulmonary Resection," The Annals of Thoracic Surgery, Vol. 87, No. 5, 2009, pp. 1539-1545. doi:10.1016/j.athoracsur.2009.02.013

[8] H. Nakamura, Y. Taniguchi, Y. Adachi, S. Fujioka and T. Haruki, "The Use of Blake Drains Following General Thoracic Surgery: Is It an Acceptable Option?" Interactive Cardio Vascular and Thoracic Surgery, Vol. 8, No. 1, 2009, pp. 58-61. doi:10.1510/icvts.2008.188086 